\title{
Review Article \\ Overview on Topical 5-ALA Photodynamic Therapy Use for Non Melanoma Skin Cancers
}

\author{
Carmen Cantisani, Giovanni Paolino, Valentina Faina, Federica Frascani, \\ Franca Cantoresi, Daniela Bianchini, Gilda Fazia, and Stefano Calvieri \\ Department of Dermatology, "Sapienza” University of Rome, Policlinico Umberto I, Viale del Policlinico 15, 00186 Rome, Italy \\ Correspondence should be addressed to Carmen Cantisani; carmencantisanister@gmail.com
}

Received 11 December 2013; Revised 9 February 2014; Accepted 15 February 2014; Published 26 March 2014

Academic Editor: Victor Loschenov

Copyright (C) 2014 Carmen Cantisani et al. This is an open access article distributed under the Creative Commons Attribution License, which permits unrestricted use, distribution, and reproduction in any medium, provided the original work is properly cited.

\begin{abstract}
Ultraviolet radiation (UV) contributes to a variety of skin diseases including inflammation, degenerative aging, and cancer. Historically, humans have been exposed to UV radiation mainly through occupational exposure; recreational UV exposure, however, has increased dramatically in recent years, because of outdoor leisure activities and to purposely tan for cosmetic purposes. Both UVB and UVA radiation have been shown to cause DNA damage and immunosuppression, the important forms of biological damage that lead to NMSC. Nonmelanoma skin cancer (NMSC) is the most common malignancy, whose public health significance is often unrecognized which continues to grow at an alarming rate, becoming an occupational disease. Available treatments alternative to surgery include photodynamic therapy, electrochemotherapy, cryotherapy, ablative lasers, 5-fluorouracil, imiquimod, ingenol mebutate, and diclofenac. Among these, photodynamic therapy is a noninvasive technique with excellent cosmetic outcome and good curative results, when used in initial stages of skin cancers for superficial lesions. It is administered under numerous and significantly varied regimens and there are a wide range of cure rates reported, permitting treatment of large and multiple lesions with excellent cosmetic results. This is an overview of photodynamic applications especially for the treatment of NMSC, with a short focus on daylight modality.
\end{abstract}

\section{Introduction}

UV energy can be subdivided into UV-A, UV-B, and UV-C components based on electrophysical properties, with UVC photons having the shortest wavelengths $(100-280 \mathrm{~nm})$ and highest energy, UV-A having the longest (315-400 nm), but least energetic photons, and UV-B falling in between. Each component of UV can exert a variety of effects on cells, tissues, and molecules. Not surprisingly, skin cancer risk generally mirrors this geographic pattern, particularly among fair-skinned sun-sensitive persons. One of the greatest risk factors for the development of cutaneous melanoma is having a fair skin complexion, which is characterized by low levels of a UV-blocking dark pigment called eumelanin in the epidermis. Individuals with light skin pigmentation suffer comparatively more skin damage from UV, because it is relatively easy for UV rays to penetrate the epidermis to damage both keratinocytes and melanocytes in the deeper layers of the epidermis. Fair-skinned individuals are exposed to higher-realized doses of UV radiation in the skin and UVinduced mutations, which directly contribute to melanoma and other forms of skin cancer, accumulating over time. Much UV-induced pathology, including skin cancer, can be avoided by minimizing UV exposure [1].

Nonmelanoma skin cancer (NMSC) is the most common malignancy, whose public health significance is often unrecognized. They comprise more than one-third of all cancers and are increasing worldwide, causing a significant economic burden at the individual and community levels. The most common NMSCs are basal cell carcinoma (BCC) and squamous cell carcinoma (SCC), occurring at a ratio of about $4: 1$ and accounting for about $90 \%$ of all skin cancers diagnosed globally. As these cancers are not reported to cancer registries in most countries, precise statistics are generally not available. However, it is estimated that between two and three million people are diagnosed worldwide each year, with an average annual increase of $3 \%$ to $8 \%$ in white 
populations in Australia, Europe, United States, and Canada over the last 30 years. The global incidence rates vary by skin complexion and geographical region and are expected to continue to rise in the coming years, due to growing exposure to ultraviolet (UV) sunlight associated with increased sunseeking behaviors and depletion of stratospheric ozone. Phenotype characteristics, environmental exposures, and genetic predisposition appear to be risk factors for their development and progression. Early detection through established methods or newer technologies is critical for reducing both skin cancer mortality and the overall skin cancer burden $[1,2]$.

\section{Photodynamic Therapy}

Topical photodynamic therapy (PDT) broadband red light source $570-670 \mathrm{~nm}$ is acknowledged to be an effective and safe treatment for NMSCs with favorable cosmetic outcomes. PDT involves the topical application of a photosensitizer, 5-aminolevulinic acid (ALA), or its methyl ester-methyl aminolevulinate (MAL) which are precursor of the endogenous photosensitizer Protoporphyrin IX and subsequent illumination of the skin lesion with light of the appropriate wavelength. In Europe, currently, only MAL is approved for the treatment of AKs, but it is significantly more expensive than ALA and therefore ALA is being used widely off label. It is a useful nonsurgical treatment option for actinic keratosis (AK), basal cell carcinoma (BCC), and in situ squamous cell carcinoma (Bowen disease), especially at sites that are cosmetically sensitive or prone to impaired wound healing [3]. It is a rapidly evolving form of phototherapy using nontoxic light-sensitive compounds that are exposed selectively to light, whereupon they become toxic to targeted malignant and other diseased cells, and it has proven ability to kill microbial cells, including bacteria, fungi and viruses (warts and molluscum contagiosum). It is used clinically to treat a wide range of medical conditions, including acne, skin aging, psoriasis, granuloma annulare, age-related macular degeneration and malignant cancers, CTCL, and extramammary Paget disease and is recognized as a treatment strategy which is both minimally invasive and minimally toxic [47]. The combination of photosensitizer, a light source and tissue oxygen, leads to the chemical destruction of any tissues which have either selectively taken up the photosensitizer or have been locally exposed to light, with recruitment of inflammatory cells, increased immune response, and vascular compromise. Single oxygen can also destroy photosensitizing agent itself preventing further action, a process referred to as photobleaching. The wavelength of the light source needs to be appropriate for exciting the photosensitizer to produce reactive oxygen species. These reactive oxygen species generated through PDT are free radicals (Type I PDT) generated through electron abstraction or transferred from a substrate molecule and highly reactive state of oxygen known as singlet oxygen (Type II PDT). In understanding the mechanism of PDT it is important to distinguish it from other light-based and laser therapies such as laser wound healing and rejuvenation, or intense pulsed light hair removal, which do not require a photosensitizer $[3,8]$.

\section{History}

While the applicability and potential of PDT have been known for over a hundred years, the development of modern PDT has been a gradual one, involving scientific progress in the fields of photobiology and cancer biology, as well as the development of modern photonic devices, such as lasers and LEDs. John Toth, in 1981, acknowledged the "photodynamic chemical effect" of the therapy and wrote the first "white paper" branding the therapy as "Photodynamic Therapy" (PDT). PDT received even greater interest as result of Thomas Dougherty helping expand clinical trials and forming the International Photodynamic Association, in $1986[3,9]$.

\section{PDT in Ancient Medicine}

The earliest recorded treatments that exploited a photosensitizer and a light source, in this case sunlight, for medical effect can be found in ancient Egyptian and Indian sources. Annals over 3000 years report the use of topically applied vegetable and plant substances to produce photoreactions in skin and cause a repigmentation of depigmented skin lesions, as seen with vitiligo and leukoderma. The photosensitizing agents used in these ancient therapies have been characterised with modern science as belonging to the psoralen family of chemicals. Psoralens are still in use today in PDT regimes to treat a variety of skin conditions, including vitiligo, psoriasis, neurodermitis, eczema, cutaneous T-cell lymphoma, and lichen ruber planus now called "PUVA-therapy" [10].

\section{20th Century Development of PDT}

The first detailed scientific evidence that agents, photosensitive synthetic dyes, in combination with a light source and oxygen could have potential therapeutic effect was made at the turn of the 20th century in the laboratory of von Tappeiner in Munich, Germany. Historically this was a time when Germany was leading the world in the industrial synthesis of dyes.

While studying the effects of acridine on paramecia cultures, Oscar Raab, a student of von Tappeiner observed a toxic effect. Fortuitously Raab also observed that light was responsible for the killing of paramecia cultures to take place. Subsequent work in the laboratory of von Tappeiner showed that oxygen was essential for the "photodynamic action"-a term coined by von Tappeiner.

With the discovery of photodynamic effects, von Tappeiner and colleagues went on to perform the first PDT trial on patients with skin carcinoma using the photosensitizer, eosin. Out of 6 patients with a facial basal cell carcinoma, treated with a $1 \%$ eosin solution and a long-term exposure either to sunlight or to arc-lamp light, 4 patients showed total tumour resolution and a relapse-free period of 12 months.

It was only much later when Thomas Dougherty and coworkers at Roswell Park Cancer Institute, Buffalo NY, clinically tested PDT again. In 1978, they published striking results in which they treated 113 cutaneous or subcutaneous malignant tumors and observed a total or partial resolution 
of 111 tumors. The active photosensitizer used in the clinical PDT trial by Dougherty was an agent called hematoporphyrin Derivative (HpD), which was first characterized in 1960 by Lipson. In his research, Lipson wanted to find a diagnostic agent suitable for the detection of tumours in patients. With the discovery of $\mathrm{HpD}$, Lipson went onto pioneering the use of endoscopes and $\mathrm{HpD}$ fluorescence to detect tumours. As its name suggests, $\mathrm{HpD}$ is a porphyrin species derived from hematoporphyrin. Porphyrins have long been considered as suitable agents for tumour photodiagnosis and tumour PDT because cancerous cells exhibit a significantly greater uptake and affinity for porphyrins compared to normal quiescent tissues. This important observation, which underlies the success of PDT to treat cancers, had been established by a number of scientific researchers prior to the discoveries made by Lipson. In 1924, Policard first revealed the diagnostic capabilities of hematoporphyrin fluorescence when he observed that ultraviolet radiation excited red fluorescence in the sarcomas of laboratory rats. Policard hypothesized at that time that the fluorescence was associated with endogenous hematoporphyrin accumulation. In 1948, Figge with coworkers showed on laboratory animals that porphyrins exhibit a preferential affinity to rapidly dividing cells, including malignant, embryonic, and regenerative cells, and because of this, they proposed that porphyrins should be used in the treatment of cancer. Subsequently many scientific authors have repeated the observation that cancerous cells naturally accumulate porphyrins and have characterised a number of mechanisms to explain it.

$\mathrm{HpD}$, under the pharmaceutical name Photofrin, was the first PDT agent approved for clinical use in 1993 to treat a form of bladder cancer in Canada. Over the next decade, both $\mathrm{PDT}$ and the use of $\mathrm{HpD}$ received wider international attention and grew in their clinical use, and lead to the first PDT treatments to receive U.S. Food and Drug Administration approval. Of all the nations beginning to use PDT in the late 20th century, the Russians were the quickest to advance its use clinically and to make many developments. Around this time, Russian scientists also collaborated with NASA medical scientists who were looking at the use of LEDs as more suitable light sources, compared to lasers, for PDT applications. PDT has also seen considerable development in Asia. Since 1990, the Chinese have been developing specialist clinical expertise with PDT using their own domestically produced photosensitizers, derived from hematoporphyrin, and light sources. PDT in China is especially notable for the technical skill of specialists in effecting resolution of difficult to reach tumours [11].

\section{Procedure}

In order to achieve the selective destruction of the target area using PDT while leaving normal tissues untouched, either the photosensitizer can be applied locally to the target area or photosensitive targets can be locally excited with light. For instance, in the treatment of skin conditions, including acne, psoriasis, and also skin cancers, the photosensitizer can be applied topically and locally excited by a light source. In the local treatment of internal tissues and cancers, after photosensitizers have been administered intravenously, light can be delivered to the target area using endoscopes and fiber optic catheters. Photosensitizers can also target many viral and microbial species, including HIV and MRSA. Using PDT, pathogens present in samples of blood and bone marrow can be decontaminated before the samples are used further for transfusions or transplants. PDT can also eradicate a wide variety of pathogens of the skin and of the oral cavities. Given the seriousness that drug resistant pathogens have now become, there is increasing research into PDT as a new antimicrobial therapy. Compared to normal tissues, most types of cancers are especially active in both the uptake and accumulation of photosensitizer agents, which makes cancers especially vulnerable to PDT. Since photosensitizers can also have a high affinity for vascular endothelial cells the net result is both the direct tumor cell death and a shutdown of the tumor vasculature. Therefore hypoxic regions of tumors are insensitive to PDT. $\mathrm{O}_{2}$ is the predominant actor of the photokilling. PDT is intended to kill malignant cells, with apoptotic or necrotic death and autophagy. It is not entirely clear which pathways to cell death are more effective. As new photosensitizer agents are developed an examination of their localization patterns and photochemistry may aid in the selection of optimal agents for tumor eradication $[3,4,8]$.

Methyl aminolevulinate (MAL) is a porphyrin precursor approved for the treatment of actinic keratoses (AKs) of the face and scalp [12-14]. The approved protocol combines topical application of MAL to individual lesions under occlusion for 3 hours followed by $37 \mathrm{~J} / \mathrm{cm}^{2}$ of red light. PpIX is then activated by red light, resulting in damage and killing of the diseased cells. In order to simplify the treatment procedure and improve tolerability, effect of daylight exposure was observed $[3,15,16]$. Instead of fast activation of large amounts of PpIX accumulated after occlusive treatment with MAL, it was thought that continuous activation of PpIX during its development might be just as efficient. The red and blue light required to activate porphyrins are part of the daylight spectrum. PDT treatment with daylight would make the treatment much easier, as the patient would not have to come back to the clinic after $3 \mathrm{~h}$ to be illuminated but we would like to add that after almost 30 minutes under artificial light patients can go under visible light and go back home. The continuous activation of small amounts of PpIX could possibly reduce treatment-related pain, which is the only acute severe adverse effect of PDT $[4,5]$. The aim of this study was to compare conventional MAL-PDT using red light-emitting diode (LED) lamps with MAL-PDT without occlusive treatment and illumination with daylight, with the primary endpoint being complete response rates of $\mathrm{AK}$. The adverse effects of the treatments were assessed as secondary endpoints.

\section{Treatment of NMSC}

Internationally licensed for the treatment of NMSC, PDT is indicated for superficial and multiple lesions, especially in the case of multifocal lesions, unclear lesion edges, risk of keloids or surgical risk factors. Red light in BCC has a superior 
penetration compared to blue and green, although experience is limited. Bowen disease has an effective result with good cosmetic results as effective as 5-FU, imiquimod 5\%, with fewer side effects, as BCCs; nodular BCC $<2 \mathrm{~mm}$ can be effective, while insufficient evidence after SCC, which can have Greater results with imiquimod 5\%, excision and Mohs surgery. For nBCCs 3 to $6 \mathrm{~h}$ of incubation are needed; better results are seen combining different treatments, as topical treatments, $\mathrm{CO}_{2}$ laser, fractional laser, curettage, and debulking 1 week before treatment and iron chelation (CP94) to increase chelation of PpIX reducing its bioconversion to heme, which is able to reduce tumor penetration. Pigmented lesions respond poorly because of interference of melanin. Many reports showed efficacy in the treatment of erythroplasia of Queyrat $[17,18]$.

\section{Photorejuvenation}

The visible signs of photodamage are characterized by wrinkling, coarse skin texture, pigmentation alterations, telangiectases, and case actinic keratosis (AKs). Intense pulsed light (IPL) photorejuvenation has been shown to improve each of the different components of photodamaged skin. Photodynamic therapy with topical 5-aminolevulinic acid (ALA-PDT) may be made also using IPL as a light source for treatment of AK. They can therefore now be treated as part of the photorejuvenation process rather than necessitating separate topical therapy with 5-fluorouracil (5-FU) or cryotherapy $[19,20]$.

\section{Photosensitizer Licensed for Mild to Moderate AKs on Face and Scalp}

The effectiveness of PDT depends on the photosensitizer used. New photosensitizer formulations have been developed

(i) Methyl aminolevulinate licensed in combination with red light $570-670 \mathrm{~nm}$ for a total dose of $75 \mathrm{~J} / \mathrm{cm}^{2}$ (metvix/Metvixia 16\%) are not currently available in USA.

(ii) ALA licensed in combination with blue light (levulan kerastick 20\%) approved for AK is the only agent approved in USA. It is a hydrophilic, low molecular weight molecule that is absorbed readily through abnormal but not from normal keratin.

(iii) ALA Patch (Alacare, $4 \mathrm{~cm}^{2}$ containing $8 \mathrm{mg}$ ALA) is recently licensed Europe.

Once ALA is absorbed by epidermal or appendage cells, it is converted to PpIX, a potent photosensitizer. Due to of limited supplies of iron, a necessary catalyst for ferrochelatase, recipient cells are unable to complete the final stage of conversion of PpIX to heme, leading to PpIX accumulation. With short application time $(<4$ hours) PpIX is largely limited to the target site. Photosensitizer typically resolves within 24 hours after application is completed. Maximal light absorption is seen at $409 \mathrm{~nm}$, and smaller peaks occur at $509 \mathrm{~nm}, 584 \mathrm{~nm}$, and $625 \mathrm{~nm}$. FDA approved 14- to 18-hour application time; however studies have demonstrated efficacy with shorter incubation period $(1 \mathrm{~h})$, more convenient for patients and doctors. MAL is more lipophillic than ALA and improved tumor tissue specificity while it was almost similar for penetration. Except for Methyl aminolevulinate the others are new formulations for AK treatment, with different light sources. New indications (not yet approved) are for actinic cheilitis, acral AK and AK in immunosuppred patients, combination therapy for AK treatment [12, 18, 21].

\section{New Concepts for Illumination Are Being Investigated}

Both ALA and MAL lead to the production of PpIX, which displays a large peak in absorption spectra at $409 \mathrm{~nm}$, with much smaller peak at $509 \mathrm{~nm}, 584 \mathrm{~nm}$, and $625 \mathrm{~nm}$. While blue light BluU (DUSA Pharmaceuticals, Wilmington, MA) or Omnilux Blue (Photo Therapeutics Inc., Carlsbad, CA) takes advantage of the largest absorption spike at $417 \mathrm{~nm}$, it is limited by depth of penetration to about 1.5 to $2 \mathrm{~mm}$. Red light $>600 \mathrm{~nm}$ requires higher energy levels to achieve the same effect, but it is able to penetrate deeper $(8-10 \mathrm{~mm})$. Filtered red or green noncoherent light sources are commonly used in Europe, whereas in USA longer wavelength light sources include diode and pulsed dye lasers as well as intense pulsed light (IPL) $[16,18]$.

5-ALA-patch for PDT of AK self-adhesive, skin coloured ALA-Patch ( $\left.2 \mathrm{mg} / \mathrm{cm}^{2} \mathrm{ALA}\right)$ easy handling application in one step, and built-in occlusion. There was no lesion preparation on treatment session. Efficacy and side effects were comparable with other photosensitizers. Nano-emulsion-based ALA formulation (BF-200) for AK phase III study using BF200 ALA (10\% ALA in gel matrix to stabilize ALA and to improve penetration); $3 \mathrm{~h}$ of incubation, illumination with Aktilite $\left(37 \mathrm{~J} / \mathrm{cm}^{2} 50-70 \mathrm{~mW} / \mathrm{cm}^{2}\right)$, or Photodyn 750 (broad band lamps $170 \mathrm{~J} / \mathrm{cm}^{2}$ ), 1-2tx better efficacy. New concepts for illumination are being investigated such as PDT of AK using different incoherent light sources which were evaluated, ALA, or MAL PDT with LED $\left(633 \pm 3 \mathrm{~nm}, 40 \mathrm{~J} / \mathrm{cm}^{2}\right)$ versus incoherent lamp $\left(580-700 \mathrm{~nm}, 100 \mathrm{~J} / \mathrm{cm}^{2}, 160 \mathrm{~mW} / \mathrm{cm}^{2}\right)$; no difference regarding efficacy, pain, patient satisfaction, and cosmetic results was seen. MAL-PDT with red light $(633 \pm$ $3 \mathrm{~nm}, 37 \mathrm{~J} / \mathrm{cm}^{2}, 50 \mathrm{~mW} / \mathrm{cm}^{2}$ ) versus IPL (610-950 nm filtered band piece $80 \mathrm{~J} / \mathrm{cm}^{2}$ ), have no difference in term of efficacy but significantly less pain with IPL. Gold standards are incoherent broadband lamps or LEDs; more studies are needed to prove efficacy of IPL. MAL-PDT is not as effective for AK on extremities, probably due to the absence of pilosebaceous glands. PDT can be used as chemoprevention in transplanted patients [22].

\section{Limits of the Procedure}

Tumour resistance and recurrence rates after PDT are however reported to range from 10 to $45 \%$. Treatment failure in PDT is thought to be multifactorial, with insufficient tumour uptake of photosensitizer and PDT-induced suppression of cutaneous antitumour responses, potentially 
important contributors for incomplete tumour killing [23]. Antitumour immunity plays a key role in reducing skin cancer development and also in skin cancer treatment responses. Immune-suppressed organ transplant recipients have significantly higher PDT failure rates compared with immunocompetent controls. The immune suppressive effects of PDT have been reported in mice and recently in humans. At the light doses and irradiance rates in current clinical use, topical PDT causes significant suppression of delayedtype hypersensitivity responses to recall antigens and depletes Langerhans cells in both normal human skin and within BCCs. The immune effects of PDT are sensitive to changes in irradiance rate; while light delivered at a high irradiance rate (approximately $75 \mathrm{~mW} \mathrm{~cm}$ ) as in clinical practice is highly immune suppressive, delivery of the same total light dose at a lower irradiance rate $(15 \mathrm{~mW} \mathrm{~cm})$ does not cause significant immune suppression in healthy volunteers. Oxidative DNA photolesions, observed in ex vivo human skin after high, but not low irradiance rate PDT, may be a key trigger of PDT-induced immunosuppression. The main disadvantage of PDT is that it can be painful, limiting its applicability $[18,24]$, which can be due to PpIX concentration, fluence rate, wavelength, treatment area size, location, sex, photosensitizer used, skin type, age, and lesion type. Pain is the major drawback of photodynamic therapy (PDT), an otherwise effective treatment for actinic keratoses (AKs). Pain is a frequent side effect of PDT. It is largely unpredictable and a major drawback of the treatment. It varies from patient to patient and the experience of burning, stinging, and prickling sensation ranges from mild to severe. In order to find the best pain prevention, investigators have tried to identify susceptible patients. In the last decade especially, there has been a spate of articles concerned with pain and PDT. Various factors have been identified to have influence on pain, but none of them has been consistently reported in all publications. Pain during PDT is associated with AK location and size. Treatment of bigger lesions $\left(>130 \mathrm{~mm}^{2}\right)$ results in more pain than smaller ones and treatment of the face is more painful than the scalp area. It is explained by tissue hypoxia and/or singlet oxygen generation, increasing the amount of pain experienced during PDT. However, other studies support our findings that the beginning of the procedure is most painful and pain is more intensive during the first procedure than during the second [24]. Pain diminution might be explained by desensitization of nociceptors, acclimatization to the treatment situation, or decreasing levels of photosensitizer during the treatment. This would indicate that pain is related to the photodynamic process itself in a time and dose dependent fashion [25]. Usually no association between the pain and the treatment dose are observed. Though Radakovic-Fijan observed a trend toward higher doses causing more pain, the finding was not statistically significant. Photodynamic therapy (PDT) is an effective but time-consuming and often painful treatment for NMSC. Daylight is the combination of direct and diffuse sunlight outdoors during daytime [15, 25]. The spectrum of electromagnetic radiation striking the earth's atmosphere is the ultraviolet light ranging from 1000 to $380 \mathrm{~nm}$, visible light from 380 to $780 \mathrm{~nm}$, and infrared light ranging from $780 \mathrm{~nm}$ to $10^{6} \mathrm{~nm}$. Home-based daylightPDT has the potential to facilitate treatment procedure and to reduce associated pain due to continuous activation of small amounts of porphyrins. Moreover, a reduced methyl aminolevulinate (MAL) concentration may reduce associated inflammation, making the treatment more tolerable for the patients. The procedure is characterized by curettage of lesions, application of medium (20-30 SPF) sun block, or photolyase inhibitors on the whole exposed area both treated and untreated; ask the patient to wait in an artificial light place for 20-30 minutes and then go under daylight from 1.5 to 2.5 hours, or the whole day under sunlight, then wash off the cream with thermal spring water, from the treated area, apply a pain relief cream exactly after PDT, and go on twice daily for 10 to 20 days, to reduce itching and burning sensation. Home-based daylight-mediated MAL-PDT was an effective and well-tolerated treatment in sun exposed areas. Pain is the major drawback of photodynamic therapy (PDT), an otherwise effective treatment for actinic keratoses (AKs). Pain during PDT is associated with AK location and size. PDT performed following MAL application to the entire face is not only more convenient but offers the potential of preventing new AKs. In order to simplify the treatment procedure and improve tolerability, effect of daylight exposure was observed [15]. Instead of fast activation of large amounts of PpIX accumulated after occlusive treatment with MAL, it was thought that continuous activation of PpIX during its development might be just as efficient. The red and blue light required to activate porphyrins are part of the daylight spectrum. PDT treatment with daylight would make the treatment much easier, as the patient would not have to come back to the clinic after $3 \mathrm{~h}$ to be illuminated but they can just go under visible light after almost 30 minutes. The continuous activation of small amounts of PpIX could possibly reduce treatment-related pain, which is the only acute severe adverse effect of PDT. Daylight-PDT would make the treatment independent of the clinic and less painful due to the continuous activation of small amounts of porphyrins during its formation. Due to the high incidence and the potential of the AK to become invasive SCC it is important to treat all of them and that their treatment be not only effective but also uncomplicated, without side effects and with good cosmetic results [21,26]. With these results, clinicians can identify patients most likely to experience pain to provide them with timely and adequate pain-control approaches during PDT or personalize treatments according to age, gender, Fitzpatrick skin type, size, clinical grade and location of the $\mathrm{AK}$, pretreatment fluorescence intensity, and the red light dose during the ALA-PDT for AKs treated on the face and scalp. The immune suppressive effects of topical photodynamic therapy (PDT) are potential contributors to treatment failure after PDT for nonmelanoma skin cancer. Nicotinamide (vitamin B3) prevents immune suppression by ultraviolet radiation, but its effects on PDT-induced immunosuppression are unknown. While the clinical relevance of these findings is currently unknown, nicotinamide may provide an inexpensive means of preventing PDTinduced immune suppression and enhancing PDT cure rates. Oral nicotinamide is well tolerated even at high doses 
(up to approximately $3 / 5 \mathrm{~g}$ per day) with a lack of reported side effects. It can be considered as an inexpensive, convenient, and nontoxic agent for skin cancer chemoprevention [27].

New chemopreventive agents that can be used to complement sunscreens may result in decreased incidence, morbidity, and mortality of skin cancer. Therefore, further research is urgently required to find an ideal chemopreventive agent that is effective, safe, accessible, and convenient either topical or systemical.

\section{Conclusion}

NMSC, with their rapidly growing incidence, negatively influence quality of life; some of them can develop in more aggressive tumor, and therefore all of them need to be treated. Their treatment needs to be efficacious and easy to be performed. PDT is a safe and effective noninvasive treatment; MAL or ALA is an attractive, well established and approved, single day therapy for mild to moderate AK, actinic cheilitis, and superficial BCC (especially of the eyelid) on face, scalp, and some SCC $[15,24]$. PDT may be the first treatment choice for multiple $\mathrm{AK}$ or field cancerisation on face and scalp with additional photorejuvenation effects. ALA-PDT using IPL as a light source is an effective treatment for both photorejuvenation and AKs [12]. For hypertrophic lesions a keratolityc agent applied before treatment and the curettage of lesions may help in improving absorption. However the conventional type is associated with inconveniently long clinic visits and discomfort during therapy; this may ride out with daylight mediated PDT, which is a simpler and more tolerable treatment procedure; it is nearly pain-free and more pleasant for both patients and physicians, especially for sunexposed areas where there are multiple lesions, independent of the weather conditions $[15,16]$. As BCC and nodular BCC are thicker lesions than AKs, the effective daylight dose, which effectively treats $\mathrm{AK}$, might not provide enough red light to ensure effective activation of PpIX in the deeper part of the lesion, probably leaving MAL-PDT under occlusion for more than 5 hours especially on special anatomical area as periocular one and may lead to a higher local skin reaction, with consequent better results. As daylight PDT is nearly pain-free it is possible to treat larger areas than with conventional type; the clinical attendance is much shorter making the clinicians able to visit and treat more patients $[21,26]$. Further studies will be needed to understand ideal condition of daylight PDT, for instance season, time, outdoor temperature, duration, geographical condition, and kind of lesions.

\section{Conflict of Interests}

The authors declare that there is no conflict of interests regarding the publication of this paper.

\section{Acknowledgments}

The manuscript was supported by Associazione Ricerca Romana Dermatologica. We thank our patients for their cooperation. The authors would also like to acknowledge colleagues who have contributed in different aspects of this study and Mrs Sonia Tofani and Mr Carlo Drago for their contribution.

\section{References}

[1] M. A. Weinstock, "Epidemiology and UV exposure," Journal of Investigative Dermatology, vol. 133, no. E1, pp. E11-E12, 2013.

[2] T. L. Diepgen and V. Mahler, "The epidemiology of skin cancer," British Journal of Dermatology, vol. 146, supplement 61, pp. 1-6, 2002.

[3] C. A. Morton, K. E. McKenna, and L. E. Rhodes, "Guidelines for topical photodynamic therapy: update," British Journal of Dermatology, vol. 159, no. 6, pp. 1245-1266, 2008.

[4] M. R. Hamblin and T. Hasan, "Photodynamic therapy: a new antimicrobial approach to infectious disease?" Photochemical and Photobiological Sciences, vol. 3, no. 5, pp. 436-450, 2004.

[5] R. Sidi Boumédine and D. C. Roy, "Elimination of alloreactive T cells using photodynamic therapy," Cytotherapy, vol. 7, no. 2, pp. 134-143, 2005.

[6] I.-M. Stender, R. Na, H. Fogh, C. Gluud, and H. C. Wulf, "Photodynamic therapy with 5-aminolaevulinic acid or placebo for recalcitrant foot and hand warts: randomised double-blind trial," The Lancet, vol. 355, no. 9208, pp. 963-966, 2000.

[7] K. Kostović, Z. Pastar, R. Ceović, Z. B. Mokos, D. S. Buzina, and A. Stanimirović, "Photodynamic therapy in dermatology: current treatments and implications," Collegium Antropologicum, vol. 36, no. 4, pp. 1477-1481, 2012.

[8] E. Skovsen, J. W. Snyder, J. D. C. Lambert, and P. R. Ogilby, "Lifetime and diffusion of singlet oxygen in a cell," Journal of Physical Chemistry B, vol. 109, no. 18, pp. 8570-8573, 2005.

[9] Z. Huang, "A review of progress in clinical photodynamic therapy," Technology in Cancer Research and Treatment, vol. 4, no. 3, pp. 283-293, 2005.

[10] M. D. Daniell and J. S. Hill, "A history of photodynamic therapy," Australian and New Zealand Journal of Surgery, vol. 61, no. 5, pp. 340-348, 1991.

[11] R. Ackroyd, C. Kelty, N. Brown, and M. Reed, "The history of photodetection and photodynamic therapy," Photochemistry and Photobiology, vol. 74, no. 5, pp. 656-669, 2001.

[12] S. R. Wiegell, S. Fabricius, I. M. Stender et al., "A randomized, multicentre study of directed daylight exposure times of $11 / 2$ vs. $21 / 2 \mathrm{~h}$ in daylight-mediated photodynamic therapy with methyl aminolaevulinate in patients with multiple thin actinic keratoses of the face and scalp," British Journal of Dermatology, vol. 164, no. 5, pp. 1083-1090, 2011.

[13] R. L. Lipson, E. J. Baldes, and M. J. Gray, "Hematoporphyrin derivative for detection and management of cancer," Cancer, vol. 20, no. 12, pp. 2255-2257, 1967.

[14] R. L. Lipson, E. J. Baldes, and A. M. Olsen, "The use of a derivative of hematoporhyrin in tumor detection," Journal of the National Cancer Institute, vol. 26, pp. 1-11, 1961.

[15] S. R. Wiegell, M. Hædersdal, P. Eriksen, and H. C. Wulf, "Photodynamic therapy of actinic keratoses with $8 \%$ and $16 \%$ methyl aminolaevulinate and home-based daylight exposure: a double-blinded randomized clinical trial," British Journal of Dermatology, vol. 160, no. 6, pp. 1308-1314, 2009.

[16] S. R. Wiegell, H. C. Wulf, R.-M. Szeimies et al., "Daylight photodynamic therapy for actinic keratosis: an international 
consensus: International Society for Photodynamic Therapy in Dermatology Wiegell et al. Daylight-mediated PDT,' Journal of the European Academy of Dermatology and Venereology, vol. 26, no. 6, pp. 673-679, 2012.

[17] J. Chen, L. Keltner, J. Christophersen et al., "New technology for deep light distribution in tissue for phototherapy," Cancer Journal, vol. 8, no. 2, pp. 154-163, 2002.

[18] P. K. Lee and A. Kloser, "Current methods for photodynamic therapy in the US: comparison of MAL/PDT and ALA/PDT," Journal of Drugs in Dermatology, vol. 12, no. 8, pp. 925-930, 2013.

[19] K. Negishi, Y. Tezuka, N. Kushikata, and S. Wakamatsu, "Photorejuvenation for Asian skin by intense pulsed light," Dermatologic Surgery, vol. 27, no. 7, pp. 627-632, 2001.

[20] P. H. Bitter Jr., "Noninvasive rejuvenation of photodamaged skin using serial, full-face intense pulsed light treatments," Dermatologic Surgery, vol. 26, no. 9, pp. 835-843, 2000.

[21] S. R. Wiegell, S. Fabricius, J. Heydenreich et al., "Weather conditions and daylight-mediated photodynamic therapy: protoporphyrin IX-weighted daylight doses measured in six geographical locations," British Journal of Dermatology, vol. 168, no. 1, pp. 186-191, 2013.

[22] T. Dirschka, P. Radny, R. Dominicus et al., "Long term (6 and 12 months) followup of two prospective, randomized, controlled phase IIItrials of photodynamic therapy with BF-200 ALA and methyl aminolaevulinate for the treatmentof actinic keratosis," British Journal of Dermatology, vol. 168, no. 4, pp. 825-836, 2013.

[23] Y. J. Matthews and D. L. Damian, “Topical photodynamic therapy is immunosuppressive in humans," British Journal of Dermatology, vol. 162, no. 3, pp. 637-641, 2010.

[24] I. M. Miller, J. S. Nielsen, S. Lophaven, and G. B. E. Jemec, "Factors related to pain during routine photodynamic therapy: a descriptive study of 301 patients," Journal of the European Academy of Dermatology and Venereology, vol. 25, no. 11, pp. 1275-1281, 2011.

[25] S. R. Wiegell, M. Hædersdal, and H. C. Wulf, "Cold water and pauses in illumination reduces pain during photodynamic therapy: a randomized clinical study," Acta Dermato-Venereologica, vol. 89, no. 2, pp. 145-149, 2009.

[26] S. R. Wiegell, M. Hædersdal, P. A. Philipsen, P. Eriksen, C. D. Enk, and H. C. Wulf, "Continuous activation of PpIX by daylight is as effective as and less painful than conventional photodynamic therapy for actinic keratoses; a randomized, controlled, single-blinded study," British Journal of Dermatology, vol. 158, no. 4, pp. 740-746, 2008.

[27] D. Surjana, G. M. Halliday, and D. L. Damian, "Nicotinamide enhances repair of ultraviolet radiation-induced DNA damage in human keratinocytes and ex vivo skin," Carcinogenesis, vol. 34, no. 5, pp. 1144-1149, 2013. 

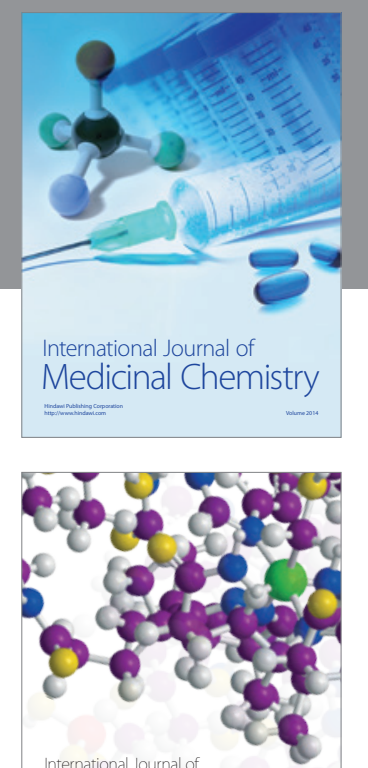

\section{Carbohydrate} Chemistry

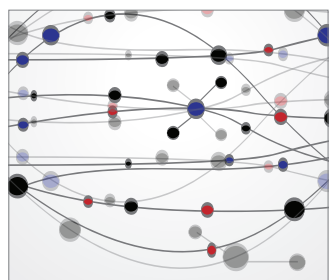

The Scientific World Journal
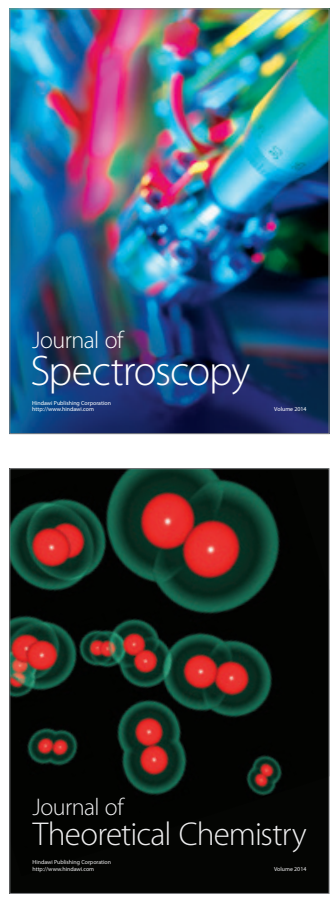
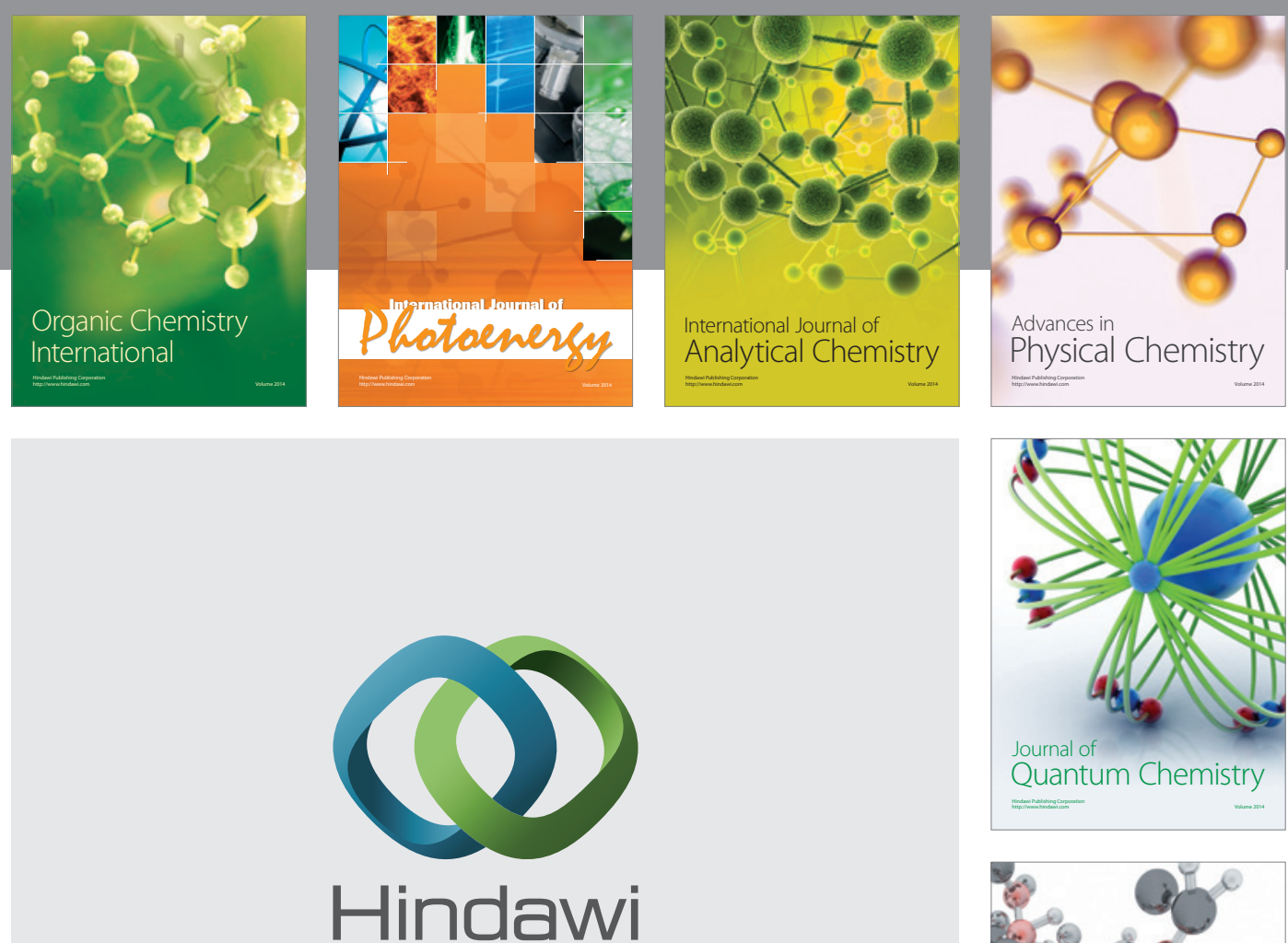

Submit your manuscripts at

http://www.hindawi.com

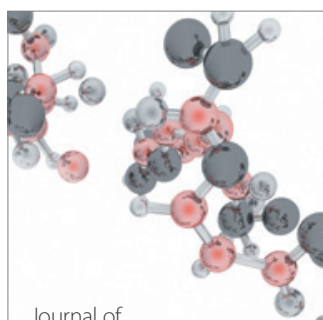

Analytical Methods

in Chemistry

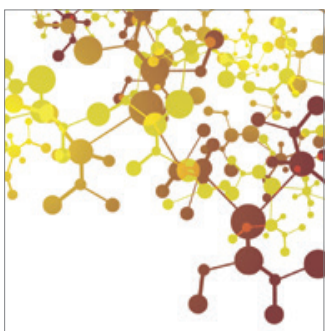

Journal of

Applied Chemistry

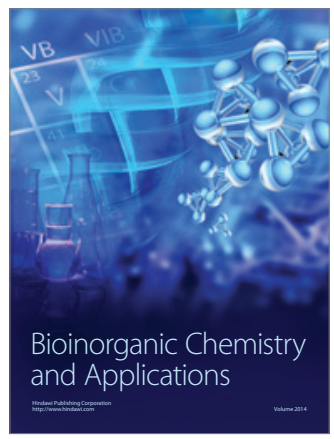

Inorganic Chemistry
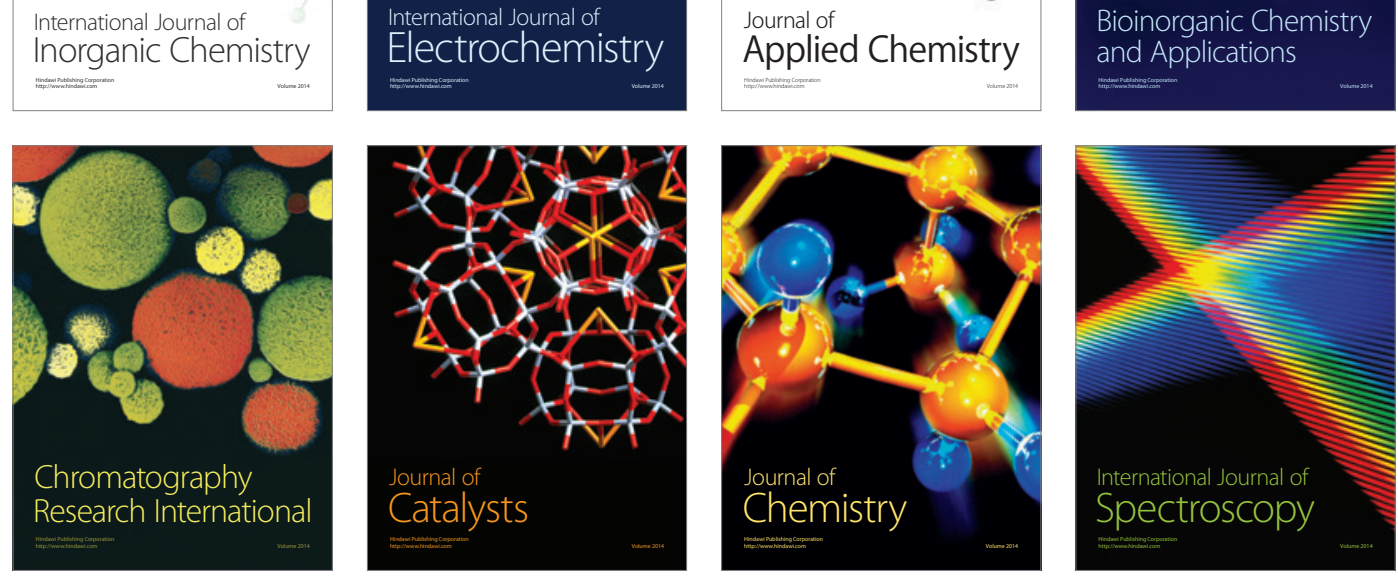